\title{
Systemic antibiotic prophylaxis for recurrent infective flares in children and adolescents with atopic dermatitis
}

\section{Dear Editor,}

Atopic dermatitis (AD) is a chronic inflammatory skin disease, affecting approximately $20 \%$ of children in Singapore. ${ }^{1}$ It is associated with skin barrier defects ${ }^{2}$ and increased skin colonisation with Staphylococcus aureus, which can trigger infective flares, especially in more severe disease. Strategies to reduce $S$. aureus colonisation and infection in AD, such as intranasal mupirocin, bleach baths and topical antiseptic agents have shown conflicting results ${ }^{3,4}$ and are not part of standard management of AD in Singapore. ${ }^{5,6}$ Literature on the use of systemic antibiotic prophylaxis in reducing infective flares of $\mathrm{AD}$ is lacking.

We performed a retrospective study of children and adolescents less than 18 years of age with moderate-tosevere $\mathrm{AD}$ and recurrent infective flares, who were prescribed prophylactic oral cephalexin twice-daily at $16-17 \mathrm{mg} / \mathrm{kg} /$ dose (maximum $500 \mathrm{mg} /$ dose), for at least 1 month. The study was approved by the SingHealth Centralised Institutional Review Board. Cases between September 2017 and April 2020 were included. Our primary outcome was to compare the number of infective flares of $\mathrm{AD}$ requiring systemic antibiotic therapy at treatment doses in the 6 months before and after starting cephalexin prophylaxis. Secondary outcomes included number of days of hospitalisation, number of unplanned clinic or emergency room visits for AD flares, number of courses of oral corticosteroid use, and number of tubes of topical corticosteroids prescribed. We also report on any adverse outcomes of cephalexin prophylaxis.

A total of 13 patients were included. The majority of patients were Chinese (84.6\%) and males (61.5\%). Most patients $(69.2 \%)$ had onset of eczema prior to schoolgoing age, with 6 patients $(46.2 \%)$ before 1 year of age, and 3 patients $(23.1 \%)$ in their preschool years (between 1 and 6 years of age). The mean age at start of cephalexin prophylaxis was 8.7 years.

Except for 1, all patients had an Investigator Global Assessment (IGA) score of 3 or 4, prior to initiation of cephalexin prophylaxis. Ten patients $(76.9 \%)$ had positive skin cultures and $3(23.1 \%)$ had negative cultures within 6 months prior to starting cephalexin prophylaxis. Of the 10 patients with positive skin cultures, 5 grew purely methicillin-sensitive $S$. aureus (MSSA) (50\%), 4 grew both MSSA and group A Streptococcus (40\%), and 1 grew methicillin-resistant $S$. aureus, MSSA and Acinetobacter (10\%). The mean duration of treatment was 152.7 days, with 5 patients $(38.5 \%)$ treated for more than 6 months. Five patients were on systemic immunosuppressants prior to initiation of cephalexin, and 1 patient was on phototherapy. None of these patients required a change in their systemic treatments during the prophylaxis period. All were continued on the same emollients and cleansers as before starting cephalexin prophylaxis.

Table 1. Comparison of patients' severity in the 6 months before and after cephalexin prophylaxis

\begin{tabular}{|c|c|c|c|c|}
\hline & $\begin{array}{l}\text { Pre-prophylaxis }{ }^{\mathrm{a}} \\
\qquad(\mathrm{n}=13)\end{array}$ & $\begin{array}{l}\text { Post-prophylaxis }^{\mathrm{a}} \\
\qquad\left(\mathrm{n}=9^{\mathrm{b}}\right)\end{array}$ & Standard deviation & $P$ value \\
\hline Infective episodes requiring systemic antibiotics & 2.8 & 0.2 & 1.72 & 1.00 \\
\hline Unplanned clinic visits & 0.6 & 0 & 2.00 & 0.63 \\
\hline Visits to children's emergency & 1.3 & 0.1 & 1.64 & 0.70 \\
\hline Hospitalisation days & 6.3 & 0.2 & 11.50 & 0.59 \\
\hline Days of systemic corticosteroids (prednisolone) & 13.4 & 3.6 & 8.08 & 0.64 \\
\hline Topical corticosteroids prescribed & 34.6 & 13.2 & 32.00 & 0.17 \\
\hline Class I & 2 & 0 & $9 / 90$ & 0.49 \\
\hline Class II & 21 & 10 & 8.91 & 0.57 \\
\hline Class III & 12 & 3 & 14.35 & 0.88 \\
\hline Class IV & 0 & 0 & 9.73 & 0.81 \\
\hline
\end{tabular}

a These measurements are means

${ }^{\mathrm{b}} 1$ patient was lost to follow-up while on cephalexin prophylaxis, 1 patient is still on cephalexin prophylaxis, and 2 patients have just completed cephalexin prophylaxis. Hence post-prophylaxis data for the 6 months post-cephalexin prophylaxis were not available for these 4 patients. 
Table 1 summarises the results of treatment. The mean number of infective flares of $\mathrm{AD}$ requiring breakthrough systemic antibiotics at treatment doses as prescribed by any physician was reduced from 2.8 to 0.2 episode in the 6 months before and after starting cephalexin prophylaxis. The mean number of days of hospitalisation and emergency visits were reduced from 6.3 to 0.2 day, and from 1.3 to 0.1 visit, respectively. Systemic corticosteroid use was reduced from 13.4 to 3.6 days, and the mean number of tubes of topical corticosteroids was reduced from 34.6 to 13.2 tubes. However, due to the small number of patients in our cohort, these results were not statistically significant. No adverse events from the use of cephalexin prophylaxis were reported.

The results of our study suggest that cephalexin prophylaxis may be a useful and safe adjunct to reduce the frequency of infective flares in patients with moderate to severe $\mathrm{AD}$. A real potential concern for cephalexin resistance should be considered against the strength of indications and benefit for prescribing it. The limitations of our study included its retrospective nature and presence of confounders such as concomitant use of other treatments, which could not be controlled for. We recommend randomised controlled trials to better evaluate the effectiveness, adverse effects, optimal dosage, and duration of oral antibiotic prophylaxis in the treatment of children and adolescents with moderateto-severe $\mathrm{AD}$ and recurrent infective flares.

\section{RERFERENCES}

1. Cheok S, Yee F, Song Ma JY, et al. Prevalence and descriptive epidemiology of atopic dermatitis and its impact on quality of life in Singapore. Br J Dermatol 2018;178:276-7.

2. Foo HL, Tey HL. A clinico-pathological approach to management of atopic dermatitis. Ann Acad Med Singap 2021;50:171-3.

3. George SMC, Karanovic S, Harrison DA, et al. Interventions to reduce staphylococcus aureus in the management of eczema. Cochrane Database of Sys Rev 2019;2019:CD003871.

4. Ewing CI, Ashcroft C, Gibbs ACC, et al. Flucloxacillin in the treatment of atopic dermatitis. Br J Dermatol 1998;138:1022-9.

5. Tay YK, Chan YC, Chandran NS, et al. Guidelines for the management of atopic dermatitis in Singapore. Ann Acad Med Singap 2016;45:439-50.

6. Tan SH, Yew YW. Editorial on Guidelines for the Management of Atopic Dermatitis in Singapore. Ann Acad Med Singap 2016; 45:437-8.

Yi Shan Ang ${ }^{1}$ MRCPCH (RCPCH, UK),

Lynette Wei Yi Wee ${ }^{1}$ MRCPCH (RCPCH, UK),

Mark Jean Aan $\underline{\operatorname{Koh}}^{1}{ }^{M R C P C H}$ (RCPCH, UK)

${ }^{1}$ Department of Dermatology, KK Women's and Children's Hospital, Singapore

Correspondence: Dr Lynette Wei Yi Wee, Department of Dermatology, KK Women's and Children's Hospital, 100 Bukit Timah Road, Singapore 229899.

Email: lynette.wee.w.y@singhealth.com.sg 